OPEN ACCESS

Check for updates

\section{Oral fluconazole use in the first trimester and risk of congenital malformations: population based cohort study}

\author{
Yanmin Zhu, ${ }^{1}$ Brian T Bateman, ${ }^{1,2}$ Kathryn J Gray, ${ }^{3}$ Sonia Hernandez-Diaz, ${ }^{4}$ Helen Mogun, ${ }^{1}$ \\ Loreen Straub, ${ }^{1}$ Krista F Huybrechts, ${ }^{1}$
}

\section{ABSTRACT}

OBJECTIVE

To examine the risk of congenital malformations associated with exposure to oral fluconazole at commonly used doses in the first trimester of pregnancy for the treatment of vulvovaginal candidiasis.

\section{DESIGN}

Population based cohort study.

\section{SETTING}

A cohort of pregnancies publicly insured in the United States, with data from the nationwide Medicaid Analytic eXtract 2000-14.

\section{PARTICIPANTS}

Pregnancies of women enrolled in Medicaid from three or more months before the last menstrual period to one month after delivery, and infants enrolled for three or more months after birth.

\section{INTERVENTIONS}

Use of fluconazole and topical azoles was established by requiring one or more prescriptions during the first trimester of pregnancy.

\section{MAIN OUTCOME MEASURES}

Risk of musculoskeletal malformations, conotruncal malformations, and oral clefts (primary outcomes), associated with exposure to oral fluconazole, diagnosed during the first 90 days after delivery, were examined.

RESULTS

The study cohort of 1969954 pregnancies included 37650 (1.9\%) pregnancies exposed to oral fluconazole and 82090 (4.2\%) pregnancies

\section{WHAT IS ALREADY KNOWN ON THIS TOPIC}

Animal studies and case reports suggest a potential teratogenic effect associated with the use of high doses of fluconazole during pregnancy

The malformations reported in case reports have a distinct phenotype, including femoral bowing, thin ribs, cleft palate, and abnormal craniofacial ossification A few controlled studies have examined the risk of congenital malformations associated with the use of fluconazole during the first trimester but findings are inconsistent

\section{WHAT THIS STUDY ADDS}

This large cohort study confirmed an increase in the risk of musculoskeletal malformations if oral fluconazole is used during the first trimester, and refuted large increased risks of conotruncal malformations, oral clefts, and other specific types of malformations

A twofold increased risk of musculoskeletal malformations was identified in the highest cumulative dose group ( $>450 \mathrm{mg}$ of fluconazole) during the first trimester Oral fluconazole during the first trimester, especially prolonged treatment at higher than commonly used doses, should be prescribed with caution, and topical azoles should be considered as an alternative treatment exposed to topical azoles during the first trimester. The risk of musculoskeletal malformations was 52.1 (95\% confidence interval 44.8 to 59.3 ) per 10000 pregnancies exposed to fluconazole versus 37.3 (33.1 to 41.4) per 10000 pregnancies exposed to topical azoles. The risks of conotruncal malformations were 9.6 (6.4 to 12.7 ) versus 8.3 (6.3 to 10.3 ) per 10000 pregnancies exposed to fluconazole and topical azoles, respectively; risks of oral clefts were 9.3 (6.2 to 12.4 ) versus 10.6 (8.4 to 12.8 ) per 10000 pregnancies, respectively. The adjusted relative risk after fine stratification of the propensity score was 1.30 (1.09 to 1.56) for musculoskeletal malformations, 1.04 (0.70 to 1.55$)$ for conotruncal malformations, and 0.91 (0.61 to 1.35 ) for oral clefts overall. Based on cumulative doses of fluconazole, the adjusted relative risks for musculoskeletal malformations, conotruncal malformations, and oral clefts overall were 1.29 (1.05 to 1.58), 1.12 (0.71 to 1.77 ), and 0.88 ( 0.55 to 1.40 ) for $150 \mathrm{mg}$ of fluconazole; 1.24 (0.93 to 1.66 ), 0.61 (0.26 to 1.39), and 1.08 ( 0.58 to 2.04 ) for more than $150 \mathrm{mg}$ up to $450 \mathrm{mg}$ of fluconazole; and 1.98 (1.23 to 3.17), 2.30 (0.93 to 5.65), and 0.94 ( 0.23 to 3.82 ) for more than $450 \mathrm{mg}$ of fluconazole, respectively.

\section{CONCLUSIONS}

Oral fluconazole use in the first trimester was not associated with oral clefts or conotruncal malformations, but an association with musculoskeletal malformations was found, corresponding to a small adjusted risk difference of about 12 incidents per 10000 exposed pregnancies overall.

\section{Introduction}

Vulvovaginal candidiasis is common in pregnant women. ${ }^{1}$ For women with uncomplicated vulvovaginal candidiasis, the recommended treatment is a short course of topical antifungal agents or one 150 mg dose of oral fluconazole. ${ }^{2}$ Although topical antifungal treatment is available over the counter in many countries, oral fluconazole is often preferred by patients because of the convenience of taking one oral dose. $^{45}$

Oral fluconazole given in the first trimester of pregnancy has steadily increased over time, from 1.1\% in 2000 to $2.6 \%$ in 2014 in the United States (eFigure 1). ${ }^{6}$ In vitro and in vivo studies in rodents suggest that high doses of fluconazole can be embryotoxic and teratogenic, causing cleft palate and skeletal abnormalities..$^{7-10}$ Case reports also suggest a potential teratogenic effect in humans associated with long term use of high dose ( $\geq 400 \mathrm{mg} /$ day) fluconazole 
during pregnancy. The malformations have a distinct phenotype, including femoral bowing, thin ribs, cleft palate, and abnormal craniofacial ossification. ${ }^{11-13}$ Several controlled studies have examined the risk of congenital malformations associated with the use of fluconazole for the treatment of vulvovaginal candidiasis during the first trimester but findings are inconsistent. ${ }^{14-19}$ Although early studies reported no increased risks of congenital malformations overall, ${ }^{14-16} 20$ more recent studies reported increased risks for specific malformations based on small numbers of women exposed to fluconazole, including a threefold increased risk of tetralogy of Fallot (seven affected infants in the exposed group), ${ }^{17}$ a sevenfold increased risk of d-transposition of the great arteries (three affected infants in the exposed group), ${ }^{18}$ and a fivefold increased risk of cleft lip with cleft palate (six affected infants in the exposed group). ${ }^{18}$ These increased risks for specific malformations have not been replicated across studies, possibly because of random variability, given the small sample size and multiple testing, ${ }^{14-18}$ residual confounding, ${ }^{14-18}$ or recall bias ${ }^{18}$ in previous studies. The risk of musculoskeletal malformations could not be estimated or was inconclusive given the wide confidence intervals in the existing literature owing to limited power. ${ }^{16} 17$ 19 The safety of fluconazole at commonly used doses for the treatment of vulvovaginal candidiasis is thus largely undefined (eTable 1).

In a large national cohort of publicly insured pregnant women, we aimed to examine the risk of congenital malformations associated with exposure to oral fluconazole at commonly used doses for the treatment of vulvovaginal candidiasis (typically 150-600 mg), with a specific focus on malformation types suggested to be associated with its use: musculoskeletal malformations, oral clefts, and conotruncal malformations (including tetralogy of Fallot and d-transposition of the great arteries).

\section{Methods}

Data source and study population

We conducted a cohort study with data from the nationwide Medicaid Analytic eXtract (MAX) from 2000 to 2014, which were the most recent data available at the time of the study. The MAX comprises a set of patient level files with information on patients' Medicaid enrollment, demographics, diagnoses, and procedures at all outpatient and inpatient settings, and dispensing records for outpatient prescription drugs for reimbursement. Within the MAX, a pregnancy cohort has been established with the family identification number shared by beneficiaries to link mothers and their infants, ${ }^{21} 22$ which has been used extensively to study the safety of drug treatments in pregnancy. ${ }^{23-28}$ We identified pregnant women aged 12-55 who were continuously enrolled in Medicaid from three or more months before the last menstrual period to one or more months after delivery, and liveborn infants enrolled from birth to three or more months afterwards, unless they died sooner. ${ }^{29}$
Exclusions were pregnancies with a chromosomal abnormality or exposure to a known teratogenic drug during the first trimester; an inpatient diagnosis of a fungal infection between 90 days before the last menstrual period (baseline) and the end of the first trimester (because inpatient treatment is not captured in MAX, which could lead to misclassification of exposure); diagnoses of oropharyngeal or esophageal candidiasis, cryptococcal meningitis, or systemic candidiasis during the baseline and first trimester (because we were interested in the safety of fluconazole at commonly used therapeutic doses for vulvovaginal candidiasis); and diagnoses of HIV infection, malignancy, or transplant during the baseline and first trimester (because fluconazole is used for prophylaxis in immunocompromised patients).

\section{Exposure and reference groups}

We identified pregnant women as exposed to fluconazole if they filled one or more prescriptions for fluconazole during the first trimester and had no dispensing for other oral antifungal agents between 90 days before the last menstrual period and the end of the first trimester. The first reference group was pregnant women who filled one or more prescriptions for topical azoles during the first trimester, with no dispensing for oral antifungal agents during baseline and the first trimester. We selected topical azoles (including butoconazole, clotrimazole, miconazole, terconazole, tioconazole, and nystatin) as a primary reference group to reduce the risk of confounding by indication and other potential unmeasured confounders. Topical azoles are considered safe owing to minimal systemic absorption and are recommended for the treatment of vulvovaginal candidiasis during pregnancy. ${ }^{1-3}$ 30-34 Pregnancies with prescriptions for oral antifungal drugs other than fluconazole were excluded because they might also be teratogenic (eTable 2). ${ }^{4} 3536$ The second reference group was women with no prescriptions for oral antifungal agents during the baseline and first trimester periods.

We further classified women exposed to fluconazole into three cumulative dose groups: $150 \mathrm{mg}$, more than $150 \mathrm{mg}$ up to $450 \mathrm{mg}$, and more than $450 \mathrm{mg}$ (during the first trimester), according to the common initial doses for the treatment of uncomplicated (one $150 \mathrm{mg}$ dose) and recurrent (100-200 mg dose for three doses) vulvovaginal candidiasis. ${ }^{23}$

\section{Congenital malformations}

The primary outcomes were musculoskeletal malformations, oral clefts, and conotruncal malformations, based on prior evidence suggesting a possible association with fluconazole. ${ }^{11-13} 17 \quad 18$ Secondary outcomes included subgroups of musculoskeletal malformations, oral clefts, and conotruncal malformations, and congenital malformations overall and cardiac malformations overall. In exploratory analyses, we also examined the risks of other organ specific malformations. 
Malformations were identified with highly specific algorithms (that is, with high positive predictive values) based on inpatient and outpatient diagnoses and procedure codes from ICD-9-CM (international classification of diseases, 9th revision, clinical modification), in the maternal and infant records within the first month and three months after delivery, respectively (eTable 3). ${ }^{37} 38$ Maternal claims within the first month after delivery were included because infant claims are sometimes recorded under the mother's name if the infant's Medicaid coverage is not activated immediately after birth. ${ }^{39}$ Starting from previously validated algorithms based on one inpatient diagnosis code (positive predictive value of $75 \%$ for cardiac malformations, $65 \%$ for musculoskeletal malformations, and 93\% for oral clefts), ${ }^{38}$ we aimed to further improve the specificity by requiring an ICD9-CM diagnosis recorded for the specific malformation on more than one date, a diagnosis on one date and a relevant surgery or procedure code, or a diagnosis on one date and death of the infant within 90 days of birth.

\section{Covariates}

Potential confounders, proxies for confounders, and risk factors for the outcome included maternal age at delivery, delivery year, race/ethnicity, region, multiple gestation, vaginal candidiasis (treatment indication), related conditions (other superficial or mucosal candidiasis, other candidiasis, other fungal or nonfungal infections, urinary tract infections), other maternal conditions (alcohol abuse or dependence, tobacco use, drug abuse or dependence, chronic renal disease, obesity or overweight, pre-existing hypertension, and diabetes), concomitant drug use (oral antibiotics, immunosuppressive agents, antidiabetic drugs, insulin, and suspected teratogens), general markers of disease burden (that is, the Obstetric Comorbidity Index), ${ }^{40}$ number of distinct prescriptions for drugs other than antifungal agents, number of distinct diagnoses, visits to the outpatient department, number of admissions to hospital, and visits to the emergency department. ${ }^{41}$ We measured vaginal candidiasis, related conditions, other maternal conditions, and concomitant drug treatments from 90 days before the last menstrual period to the end of the first trimester, and general markers of disease burden from 90 days before the last menstrual period to the last menstrual period, to avoid adjusting for intermediate variables on the causal pathway.

\section{Analyses}

We calculated absolute risks, risk differences, and relative risks with 95\% confidence intervals for each outcome. We first compared pregnancies exposed to fluconazole with pregnancies not exposed to fluconazole. We then restricted the reference group to women who filled a prescription for topical azoles during the first trimester because they are likely to be more comparable with the group exposed to fluconazole than the group not exposed (main analysis). As a further adjustment, we accounted for all covariates described above by stratification of the propensity score. We estimated the propensity score for fluconazole versus users of topical azoles with logistic regression and excluded observations from the non-overlapping regions of the propensity score distributions. Specifically, we created 50 equally sized propensity score groups based on the distribution in the pregnancies exposed to fluconazole, and weighted the pregnancies in the reference group by the distribution of the treated pregnancies in the propensity score groups in the outcome models. ${ }^{42}$ Thus extreme weights owing to propensity scores that are close to 0 or 1 are unlikely; an important strength in this circumstance where the prevalence of exposure during the first trimester is low. ${ }^{43}$ To examine the covariate balance between groups, we calculated the standardized mean differences: a value of less than 0.1 for a covariate was considered as well balanced. Relative risks and risk differences were estimated with generalized linear regression models (PROC GENMOD in SAS, SAS Institute). The same approach was used for analyses by cumulative dose. The unit of analysis was pregnancy. Accounting for correlations in mothers with multiple pregnancies with the robust variance estimator did not change the confidence intervals appreciably, and so correlation structures were omitted from the analyses.

\section{Sensitivity analyses}

We conducted sensitivity analyses to test the robustness of our findings. First, to evaluate the risk associated with treatment of uncomplicated vulvovaginal candidiasis, we redefined exposure as filling only one prescription for $150 \mathrm{mg}$ of fluconazole. Second, because patients might not consume the dispensed drugs, we required two or more fluconazole prescriptions dispensed during the first trimester, assuming that if two prescriptions were filled, the drug was more likely to be taken. Third, to evaluate the effect of potentially missing late diagnoses of outcomes, we extended follow-up of infants to one year. Fourth, as a negative control analysis, we assessed the risk of congenital malformations in women who filled their first fluconazole prescription in gestational weeks 16-28 (after the presumed etiologically relevant window). Presuming that there would be no true effect or defects if fluconazole was used in the second trimester, any association suggesting an increased risk in this analysis would be indicative of residual confounding. The propensity score was re-estimated in all sensitivity analyses that affected the definition of exposure. Finally, because the cohort included live births only, we quantified the potential impact of differential pregnancy losses in the fluconazole and topical azoles groups within levels of covariates with methods described previously (eTable 15). ${ }^{23}$

\section{Patient and public involvement}

No patients were involved in setting the research question or the outcome measures, nor were they involved in developing plans for design or 
implementation of the study. No patients were asked to advise on interpretation or writing up of results. There are no plans to disseminate the results of the research to study participants or the relevant patient community.

\section{Results}

The cohort of 1969954 pregnancies (1.9\% multiple pregnancies) included 37650 (1.9\%) pregnancies exposed to oral fluconazole and 82090 (4.2\%) pregnancies exposed to topical azoles during the first trimester (eTable 2). Compared with pregnancies not exposed to fluconazole, women in the fluconazole group were more likely to be black, have a diagnosis of vulvovaginal candidiasis and other infections, be overweight or obese and have pre-existing hypertension and diabetes, use other drugs, and use healthcare facilities more often. Patient characteristics between the fluconazole group and the topical azole groups were more similar (including vulvovaginal candidiasis, related conditions, other comorbidities, concomitant drug treatments, and healthcare use) than those between the fluconazole group and the unexposed group. After weighting of the propensity score within each group, prespecified covariates were well balanced between the groups, with a standardized difference of less than 0.1 for all covariates (table 1 ).

\section{Risk of congenital malformations}

The risk of musculoskeletal malformations was 52.1 (95\% confidence interval 44.8 to 59.3 ) per 10000 pregnancies exposed to fluconazole versus 38.0 (37.1 to 38.9) per 10000 pregnancies not exposed to fluconazole, corresponding to an unadjusted relative risk of 1.37 (1.19 to 1.58). The risk in pregnancies exposed to topical azoles was 37.3 (33.1 to 41.4) per 10000 pregnancies. Comparing oral fluconazole with topical azoles resulted in an unadjusted relative risk of 1.40 (1.17 to 1.67). After adjustment for all confounding variables, the relative risk compared with topical azoles was 1.30 (1.09 to 1.56) and the risk difference was 12.15 (3.69 to 20.60) per 10000 pregnancies.

The risk of conotruncal malformations was 9.6 (95\% confidence interval 6.4 to 12.7 ) per 10000 pregnancies exposed to fluconazole, 7.5 (7.1 to 7.9) per 10000 pregnancies not exposed to fluconazole, and 8.3 (6.3 to 10.3 ) per 10000 pregnancies exposed to topical azoles. The unadjusted relative risk for use of fluconazole was 1.27 (0.91 to 1.77$)$ versus pregnancies not exposed, and 1.15 (0.77 to 1.73) versus pregnancies exposed to topical azoles. The adjusted relative risk versus exposed to topical azoles was 1.04 ( 0.70 to 1.55 ), corresponding to an adjusted risk difference of 0.37 ( -3.40 to 4.12$)$ per 10000 pregnancies.

For oral clefts, the absolute risks were $9.3(95 \%$ confidence interval 6.2 to 12.4 ), 11.8 (11.3 to 12.2), and 10.6 (8.4 to 12.8 ) per 10000 pregnancies exposed to fluconazole, not exposed to fluconazole, and exposed to topical azoles, respectively. The unadjusted relative risk versus pregnancies not exposed to fluconazole was 0.79 (0.57 to 1.10$)$. The relative risks versus pregnancies exposed to topical azoles were 0.88 (0.59 to 1.30) in unadjusted analyses and 0.91 (0.61 to 1.35) in adjusted analyses, with an adjusted risk difference of -0.90 ( -4.70 to 2.88 ) per 10000 pregnancies (table 2, fig 1, fig 2, and eTable 5).

For the secondary outcomes, the adjusted relative risks versus topical azoles were 1.00 (0.94 to 1.06) for congenital malformations overall and 0.98 (0.87 to 1.10) for cardiovascular malformations overall. The numbers in the musculoskeletal malformation subgroups for pregnancies exposed to fluconazole were small $(<11)$ for five out of the eight subgroups. The adjusted relative risk estimates versus topical azoles were increased for all subgroups except for other anomalies of the ribs and sternum, ranging from 1.12 to 4.29 , although the associations were imprecisely estimated. The adjusted relative risk was 0.79 (0.46 to 1.35) for tetralogy of Fallot and 2.01 (1.08 to 3.75) for transposition of great vessels based on 19 infants in the group exposed to fluconazole. The adjusted relative risks versus topical azoles for cleft palate, cleft lip, or cleft palate with cleft lip were 0.97 (0.56 to 1.69), 1.23 (0.64 to 2.37 ), and 0.83 (0.44 to 1.58 ), respectively (fig 2 and eTable 5).

In exploratory analyses, the other organ specific malformations generally did not show a strong increased risk although the confidence intervals for some were wide because of limited numbers (eTable 5).

\section{Analyses according to fluconazole dose groups}

For pregnancies exposed to fluconazole, 24755 $(65.8 \%)$ had a cumulative dose of $150 \mathrm{mg}, 10416$ (27.7\%) had a dose of more than $150 \mathrm{mg}$ up to 450 $\mathrm{mg}$, and 2109 (5.6\%) had a dose of more than $450 \mathrm{mg}$ (highest cumulative dose included: $6000 \mathrm{mg}$ ) during the first trimester. Compared with topical azoles, the increase in the risk of musculoskeletal malformations was greatest for the group of pregnancies with a cumulative dose of more than $450 \mathrm{mg}$ (adjusted relative risk 1.98 (95\% confidence interval 1.23 to 3.17) for the $>450 \mathrm{mg}$ group, 1.29 (1.05 to 1.58) for the $150 \mathrm{mg}$ group, and 1.24 (0.93 to 1.66$)$ for the $>150-450 \mathrm{mg}$ group). For conotruncal malformations, with fewer than 11 exposed pregnancies in the more than $450 \mathrm{mg}$ dose group, the relative risk was 2.30 (0.93 to 5.65) compared with topical azoles. The upper limit of the $95 \%$ confidence interval corresponded to an adjusted risk difference of 34 incidents per 10000 exposed pregnancies. In contrast, for oral clefts, no evidence of an increased risk was found in the group of pregnancies with a cumulative dose of more than $450 \mathrm{mg}$ (fig 3, fig 4, eTable 9). A higher risk for secondary and exploratory outcomes was not found for pregnancies in the groups with higher doses of fluconazole, although the data were sparse (eTable 9).

\section{Sensitivity analyses}

After fine stratification weighting of the propensity score, prespecified covariates were well balanced 


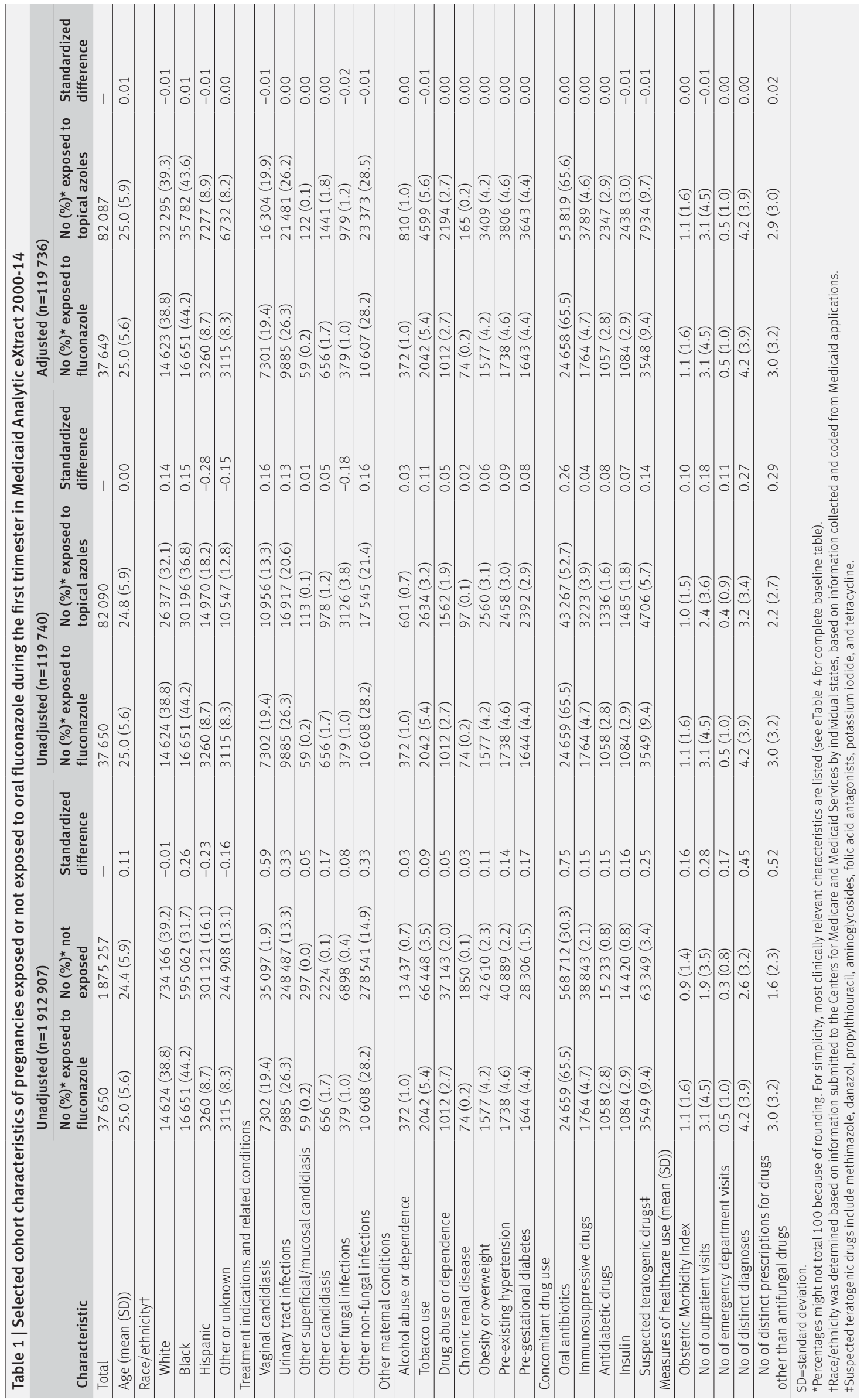




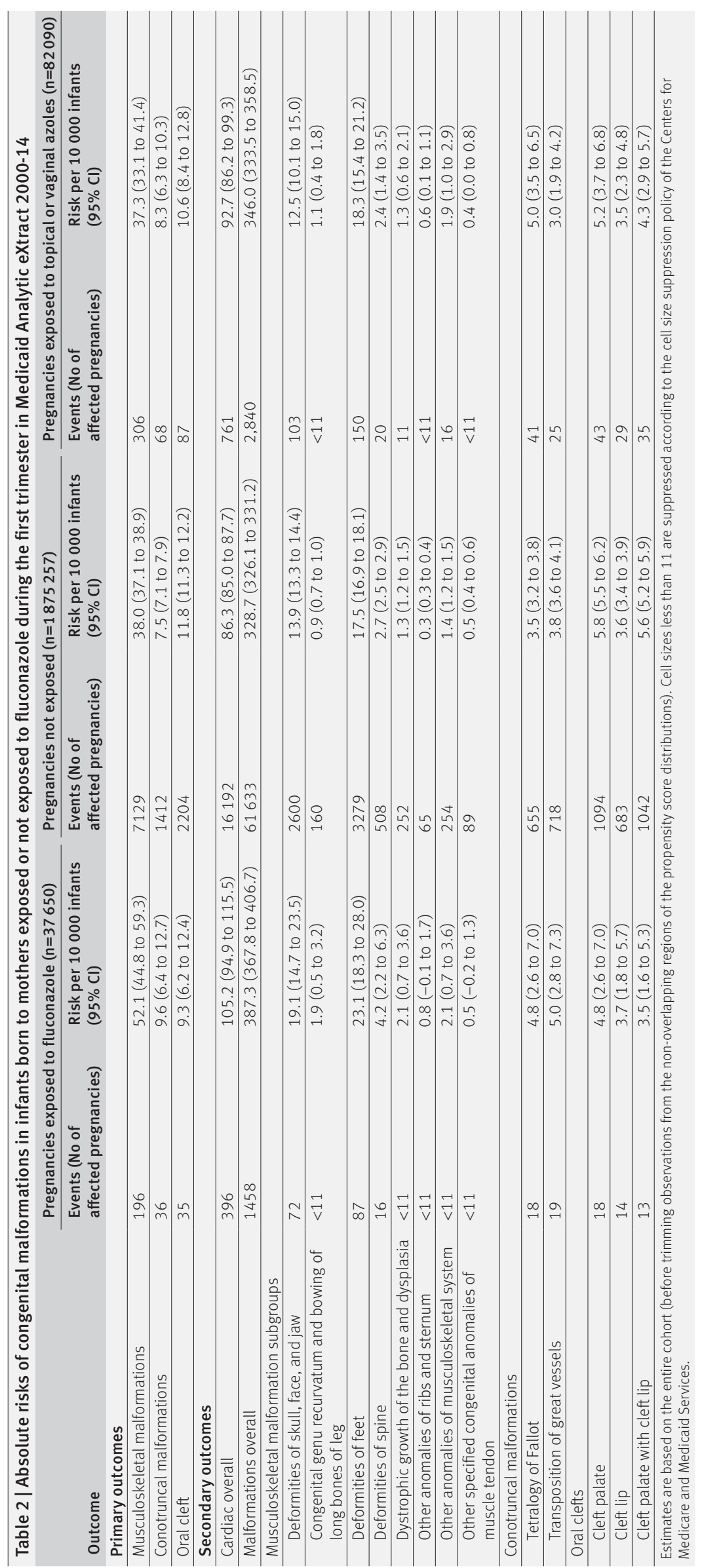

between the groups in all of the sensitivity analyses (eTables 4 and eTables 6-8). The results of restricting the fluconazole group to pregnancies with one 150 mg prescription were consistent with the main analyses. Associations did not strengthen with two or more prescriptions. The overall findings were not affected when we determined the outcomes during the first year of life. In the negative control analyses, we no longer observed an increased risk for musculoskeletal malformations, or an increased risk for the other primary or secondary outcomes, except for transposition of great vessels (fig 3, fig 4, and eTables 10-14). The absolute risk of transposition of great vessels in the topical azole group was lower than that in the group not exposed (3.0 (95\% confidence interval 1.9 to 4.2 ) v 3.8 (3.6 to 4.1) per 10000 pregnancies, table 2). We therefore observed an increased incidence of the transposition of great vessels for the group exposed to fluconazole relative to the group exposed to topical azoles in the main analyses and in the negative control analyses but not in the group who were not exposed (eTable 5 and eTable 12).

In the most extreme scenario that we considered, to quantify the impact of selection bias owing to restriction to live births, where we assumed at least $45 \%$ nonlive births in infants with malformations exposed to topical azoles and 65\% non-live births in infants with malformations exposed to oral fluconazole, the relative risk estimate for musculoskeletal malformations would shift from 1.30 to 1.50 (eFigure 2), whereas the relative risk would remain below 1.25 for conotruncal malformations (eFigure 3) and oral clefts (eFigure 4).

\section{Discussion \\ Principal findings}

In this large nationwide cohort of pregnant women insured by Medicaid, we found no association between oral fluconazole use overall during the first trimester of pregnancy at common therapeutic doses and the risk of conotruncal malformations or oral clefts after controlling for potential confounding. We observed an increased risk of musculoskeletal malformations, however, with a 30\% increased risk at cumulative doses of $150 \mathrm{mg}$ and cumulative doses of more than $150 \mathrm{mg}$ up to $450 \mathrm{mg}$, and an almost twofold increased risk for cumulative doses more than $450 \mathrm{mg}$, corresponding to about 20 and 80 excess incidents, respectively, per 10000 live births exposed to fluconazole in the womb, based on the upper limit of the $95 \%$ confidence interval.

\section{Comparison with other studies}

Case reports have suggested a distinct phenotype of congenital malformations attributable to exposure to high doses (400-800 mg/day) of fluconazole prenatally. ${ }^{11-13} 44$ In 2011, the United States Food and Drug Administration (FDA) issued a drug safety communication about fluconazole at high doses for indications other than vulvovaginal candidiasis and birth defects, highlighting the lack of well controlled studies examining the risk of one $150 \mathrm{mg}$ dose for 


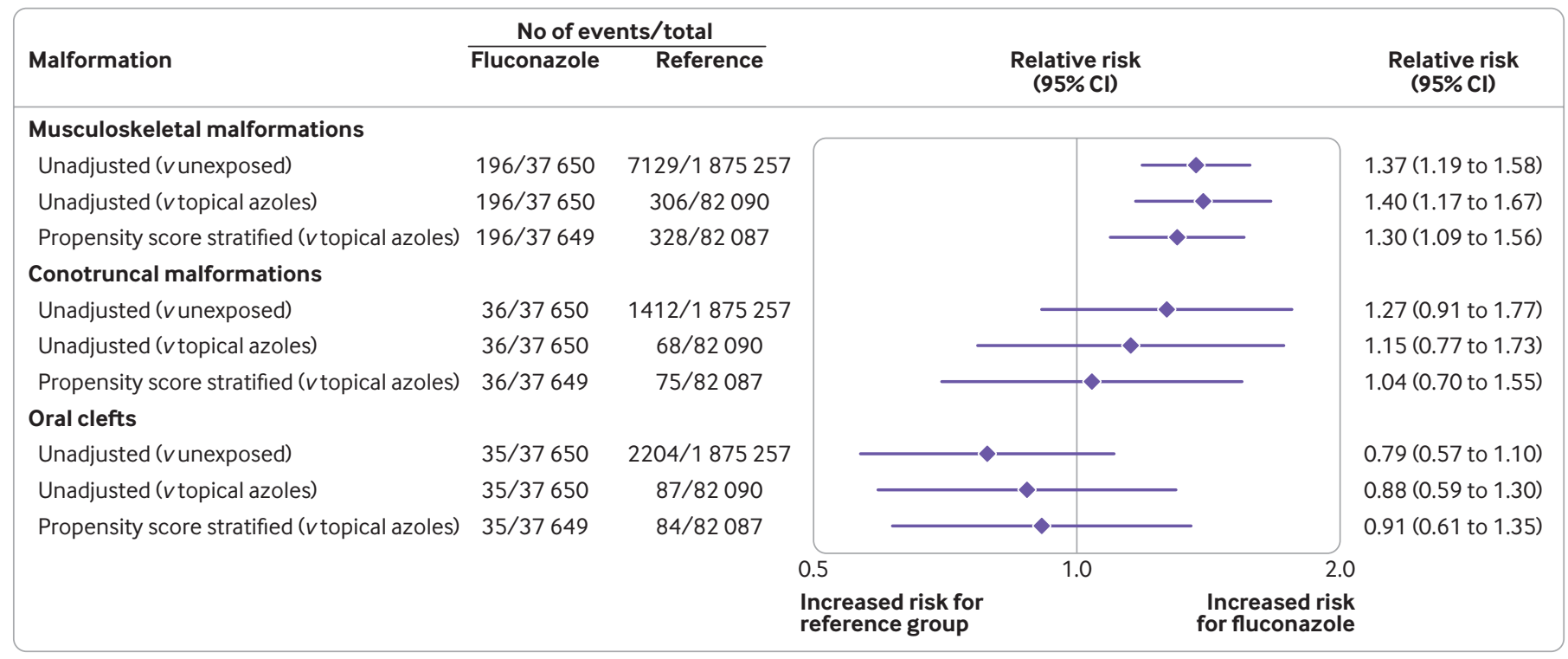

Fig 1 | Risk of congenital malformations in infants after exposure to fluconazole during the first trimester in Medicaid Analytic eXtract 2000-14: primary outcomes in main analyses. Accounting for correlations within mothers with multiple pregnancies using robust variance estimator did not change the confidence intervals appreciably, and so correlation structures were omitted from all analyses

vulvovaginal candidiasis and the limited power of existing studies to detect an increase in the risk of rare birth defects (eTable 1, studies 1-3). ${ }^{44-16} 45$ Whether exposure to fluconazole at common therapeutic doses for vulvovaginal candidiasis led to an increased risk of specific congenital malformations was unclear.

The increased risk of musculoskeletal malformations, that we observed, associated with exposure to fluconazole at common therapeutic doses during the first trimester, aligns with the patterns of malformations reported in previous case reports at high doses, summarized by the FDA. ${ }^{11-13}$ Studies in rodent embryos showed that exposure to fluconazole was associated with fusion of the branchial arches and hypoplasia of the first or second branchial arch, a series of externally visible anterior tissue bands under the early brain that give rise to the craniofacial skeletal structures and musculature. ${ }^{8} 9$ Previous studies might have been unable to detect this increased risk, probably because of an insufficient sample size. We also observed an almost twofold increased risk in the higher dose group (>450 mg of fluconazole), consistent with the dose-response relation in animal models. ${ }^{736}$

Since the announcement by the FDA, a few studies have reported signals on the teratogenicity of exposure to fluconazole at common therapeutic doses (eTable 1, studies 4-6). ${ }^{17-19}$ A Danish cohort study reported a threefold increased risk of tetralogy of Fallot based on seven affected infants who were exposed to fluconazole during the first trimester. ${ }^{17}$ In our study, the number of pregnancies exposed to fluconazole was five times greater, reducing the likelihood of a chance finding. With an active comparator group, adjusting for a broad range of confounding variables, and restricting the cohort to pregnancies with no exposure to other oral antifungal drugs during the baseline or first trimester, we reduced the potential for residual confounding. A case-control study reported a fivefold increased risk of oral clefts (six affected infants exposed to fluconazole during the first trimester $)^{18}$; however, limited control of confounding could have contributed to this finding. They also reported a sevenfold unadjusted increased risk for d-transposition of the great arteries (three affected infants exposed to fluconazole during the first trimester). ${ }^{18}$ We observed no increased risk compared with pregnancies not exposed to fluconazole, but a twofold increased risk versus exposure to topical azoles, which was likely a chance finding because of a lower than expected risk of the outcome in users of topical azoles. Another study reported an increased risk of cardiac septal closure anomalies identified based on one or more diagnosis codes for pregnancies exposed to a cumulative dose of more than 150 $\mathrm{mg}$ of fluconazole (13 affected infants exposed to fluconazole during the first trimester). ${ }^{19}$ In contrast, we did not see an increased risk for secundum atrial septal defect, atrioventricular septal defect, or ventricular septal defect across dose groups and a broad range of analyses with highly specific outcome definitions.

\section{Strengths of the study}

Strengths of this study include the large sample size, which allowed us to examine the risk of rare malformations that have been suggested to be associated with fluconazole and specific dose groups; use of highly specific validated algorithms to define outcomes ${ }^{38}$; use of pharmacy prescriptions to calculate exposure, avoiding recall bias; use of an active comparator group to reduce confounding by indication and other potential unmeasured confounders, and generate evidence more applicable to clinical decision making; and careful control for a broad range of potential confounding variables. 


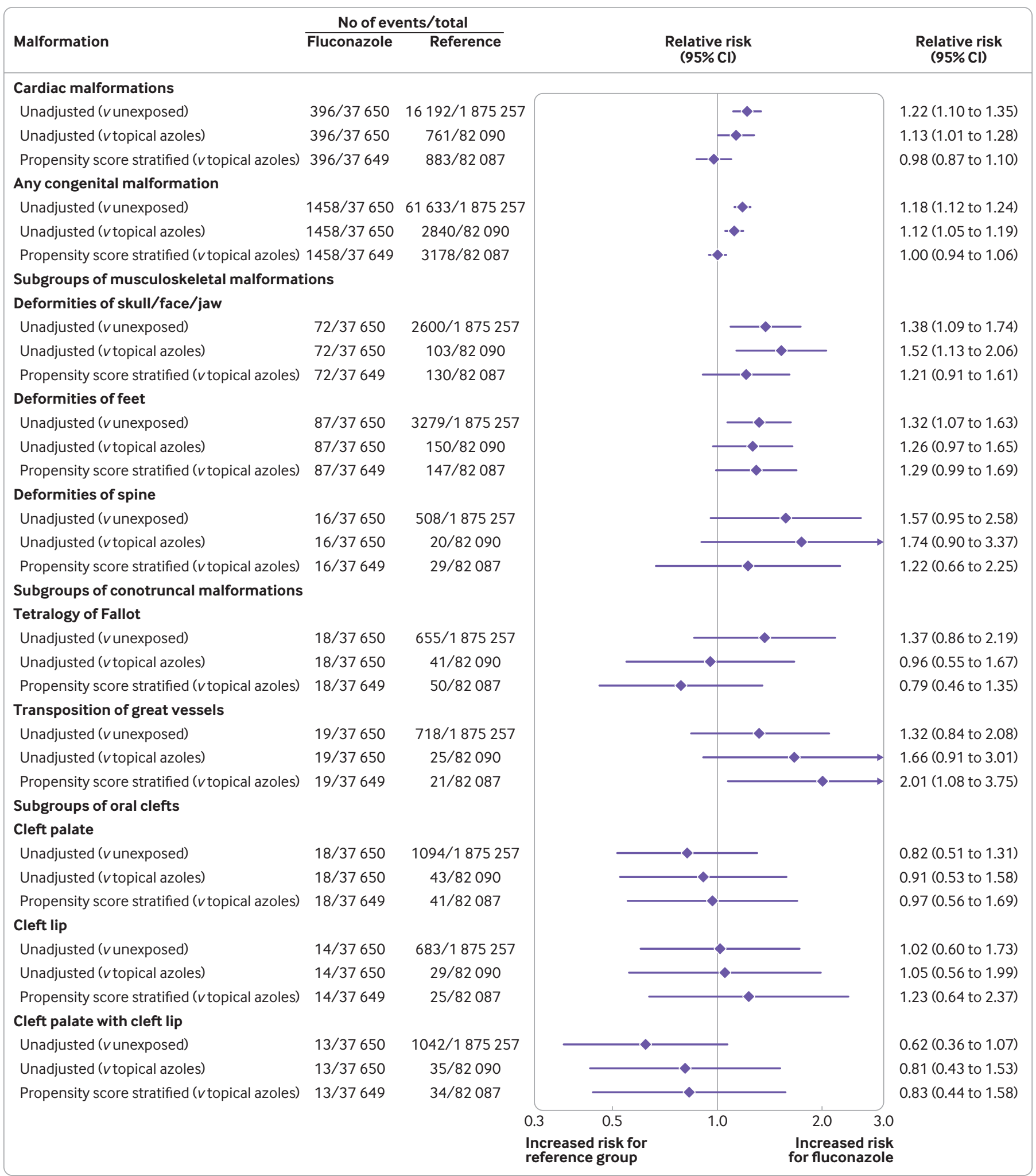

Fig 2 | Risk of congenital malformations in infants after exposure to fluconazole during the first trimester in Medicaid Analytic eXtract 2000-14: secondary outcomes in main analyses. Results of other subgroups of musculoskeletal malformations with less than 11 outcomes in pregnancies exposed to fluconazole are presented in eTable 5. Accounting for correlations within mothers with multiple pregnancies using robust variance estimator did not change the confidence intervals appreciably, and so correlation structures were omitted from all analyses

Limitations of the study

Despite the large study size, the number of outcomes for the least common malformations was relatively low, particularly in the subgroup analyses. Also, we were not able to determine if the women consumed the dispensed drug. We therefore conducted sensitivity analyses requiring two or more prescription fills, which increased the likelihood that the drug was consumed. 


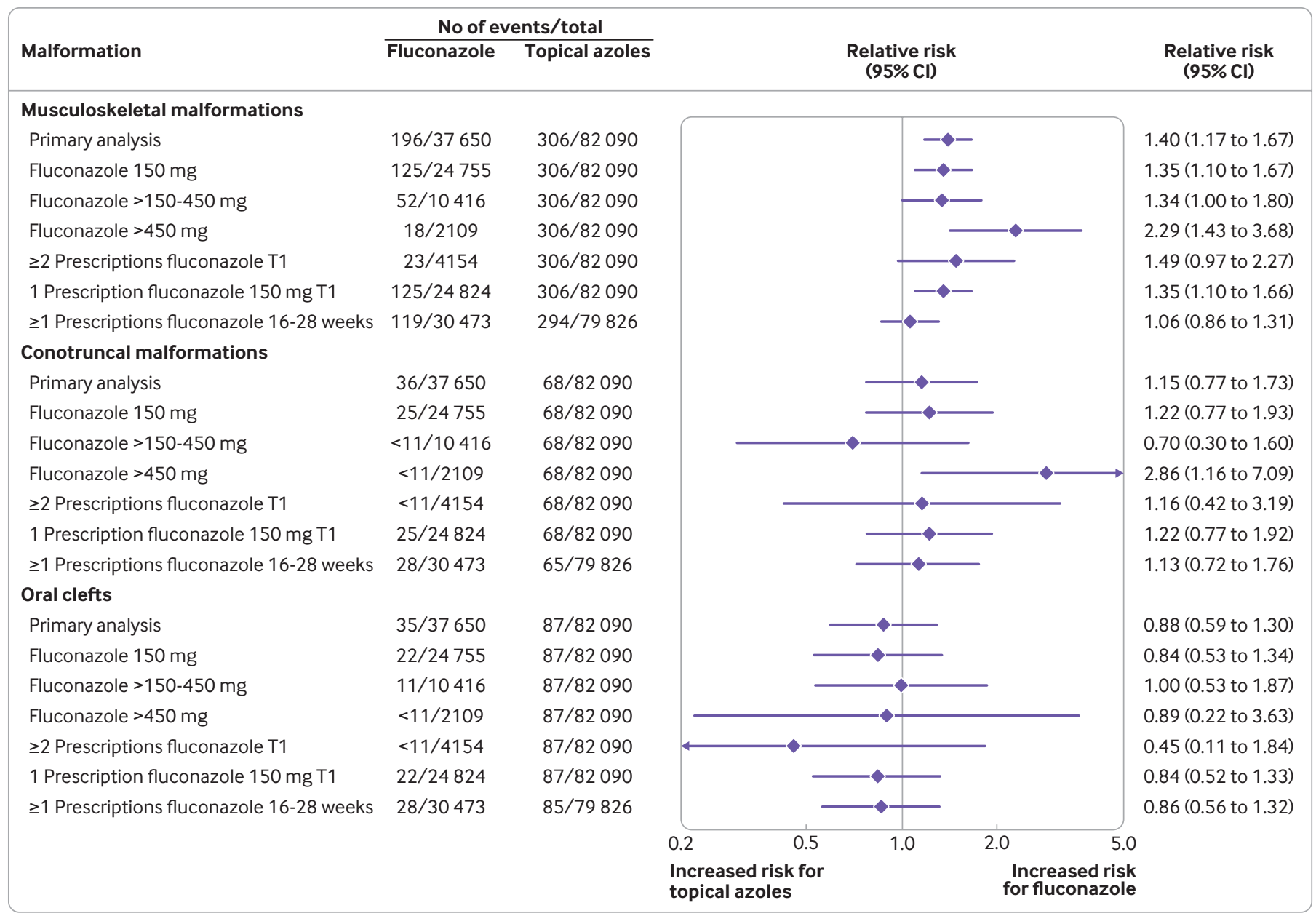

Fig 3 | Risk of congenital malformations in infants after exposure to fluconazole during the first trimester in Medicaid Analytic eXtract 2000-14: unadjusted associations in sensitivity analyses. Cell sizes less than 11 are suppressed according to the cell size suppression policy of the Centers for Medicare and Medicaid Services. Accounting for correlations within mothers with multiple pregnancies using robust variance estimator did not change the confidence intervals appreciably, and so correlation structures were omitted from all analyses

The results were consistent with those from the main analyses. Another limitation was potential residual confounding by unmeasured or poorly measured variables (eg, obesity or overweight) that could account for the increased risk. To deal with this concern, we conducted a negative control analysis. If we still observed an increased risk with the use of fluconazole after the etiologically relevant time window, it would be due to confounding rather than a true causal association. This analysis yielded no increased risk of musculoskeletal malformations, suggesting that the association with exposure in the first trimester was likely to be causal. Also, the cohort who were insured by Medicaid tended to be younger, racially diverse, and had a high burden of disabilities. These characteristics, however, are unlikely to affect the biological relations examined and so the results should be generalizable. Our cohort was restricted to live births and therefore severe cardiac malformations resulting in spontaneous abortions, stillbirths, or terminations of pregnancy for cardiac anomalies would be missed. Under the most extreme scenario, the increased risks of conotruncal malformations and oral clefts would be minimal, whereas the risk for musculoskeletal malformations would be slightly higher than the estimate in the primary analyses.

\section{Conclusions}

Oral fluconazole is the firstline treatment for vulvovaginal candidiasis and is commonly used in women of reproductive age, leading to prenatal exposure. $^{1-3} 30$ The safe use of fluconazole during pregnancy has been controversial because of the conflicting reports of teratogenicity and the limited data on the low dose treatment for vulvovaginal candidiasis. Our study confirmed an increase in the risk of musculoskeletal malformations if fluconazole is used during the first trimester, and refuted large increased risks of conotruncal malformations, oral clefts, and other specific types of malformations, although the point estimate for conotruncal malformations was increased at higher doses (>450 mg cumulative dose), corresponding to a small adjusted risk difference of no more than 34 incidents per 10000 exposed pregnancies. Oral fluconazole during the first trimester, especially prolonged treatment at higher than commonly used doses, should be prescribed with caution, and 


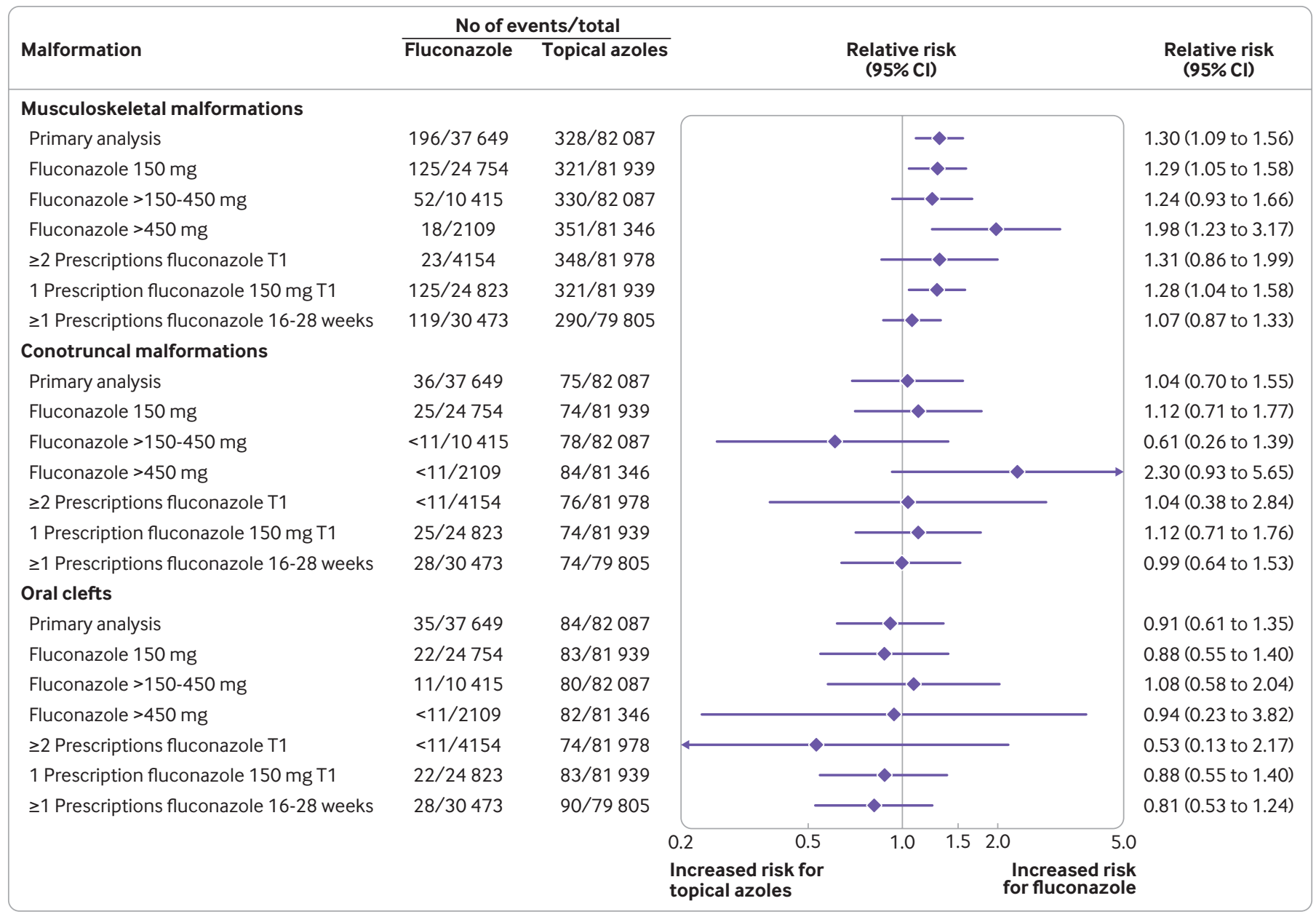

Fig 4 | Risk of congenital malformations in infants after exposure to fluconazole during the first trimester in Medicaid Analytic eXtract 2000-14: adjusted associations in sensitivity analyses. Cell sizes less than 11 are suppressed according to the cell size suppression policy of the Centers for Medicare and Medicaid Services. Accounting for correlations within mothers with multiple pregnancies using robust variance estimator did not change the confidence intervals appreciably, and so correlation structures were omitted from all analyses

topical azoles should be considered as an alternative treatment.

Contributors: YZ conceptualized and designed the study, did the analyses, and drafted the initial manuscript. KFH, BTB, and SH-D conceptualized and designed the study, critically reviewed the results of analyses, and reviewed and revised the manuscript. HM did the analyses and reviewed and revised the manuscript. KJG and LS provided input to the study concept and design, critically reviewed the results of analyses, and reviewed and revised the manuscript. All authors approved the final manuscript as submitted. The corresponding author attests that all listed authors meet authorship criteria and that no others meeting the criteria have been omitted. YZ is the guarantor.

Funding: This study was funded by the Division of

Pharmacoepidemiology and Pharmacoeconomics, Department of Medicine, Brigham and Women's Hospital. KJG was supported by the K12 BIRCWH career development award from the Office of Research on Women's Health to Harvard Medical School. The funders had no role in considering the study design or in the collection, analysis, interpretation of the data, writing of the report, or decision to submit the article for publication.

Competing interests: All authors have completed the ICMJE uniform disclosure form at www.icmje.org/coi_disclosure.pdf and declare: support from the Division of Pharmacoepidemiology and Pharmacoeconomics, Department of Medicine, Brigham and Women's Hospital for the submitted work. KFH reports grants from the National Institutes of Health during the conduct of the study and grants to her institution from Eli Lilly, GlaxoSmithKline, and Boehringer Ingelheim outside the submitted work. BTB reports grants from the National
Institutes of Health during the conduct of the study and grants to his institution from Eli Lilly, GlaxoSmithKline, Baxalta, Pacira, and Pfizer outside the submitted work. He received consulting fees from Merck for Mothers, Aetion, and the Alosa Foundation. KJG reports being a consultant and educational speaker on expanded carrier screening and preterm birth for Quest Diagnostics and served on an advisory panel for Illumina on the prediction of adverse pregnancy outcomes, and personal fees from BillionToOne. SH-D reports grants from the National Institutes of Health during the conduct of the study; grants to her institution from Lilly, Takeda, Pfizer, and GlaxoSmithKline and personal fees from UCB outside the submitted work; and being an advisor for the Antipsychotics Pregnancy Registry and epidemiologist for the North American Antiepileptics Pregnancy Registry, both at Massachusetts General Hospital. The remaining authors declare no conflicts of interest.

Ethical approval: The research was approved by the institutional review board of Brigham and Women's Hospital (IRB 2011P002580), which granted a waiver of informed consent.

Data sharing: No additional data available.

The lead author affirms that the manuscript is an honest, accurate, and transparent account of the study being reported; that no important aspects of the study have been omitted; and that any discrepancies from the study as planned (and, if relevant, registered) have been explained.

Dissemination to participants and related patient and public communities: There are no plans to disseminate the results of the research to study participants or the relevant patient community. Owing to restricted access to the data, the authors used the existing database to conduct the research and had no direct contact 
information of the study participants to disseminate the results of the research.

This is an Open Access article distributed in accordance with the Creative Commons Attribution Non Commercial (CC BY-NC 4.0) license, which permits others to distribute, remix, adapt, build upon this work non-commercially, and license their derivative works on different terms, provided the original work is properly cited and the use is noncommercial. See: http://creativecommons.org/licenses/by-nc/4.0/.

1 Aguin TJ, Sobel JD. Vulvovaginal candidiasis in pregnancy. Curr Infect Dis Rep 2015;17:462. doi:10.1007/s11908-015-0462-0

2 Pappas PG, Kauffman CA, Andes DR, et al. Clinical practice guideline for the management of candidiasis: 2016 update by the Infectious Diseases Society of America. Clin Infect Dis 2016;62:e1-50. doi:10.1093/cid/civ1194

3 Workowski KA, Bolan GA, Centers for Disease Control and Prevention. Sexually transmitted diseases treatment guidelines, 2015. MMWR Recomm Rep 2015;64(RR-03):1-137.

4 Pilmis B, Jullien V, Sobel J, Lecuit M, Lortholary O, Charlier C. Antifungal drugs during pregnancy: an updated review. J Antimicrob Chemother 2015:70:14-22 doi:10.1093/jac/dku355

5 Sobel JD. Factors involved in patient choice of oral or vaginal treatment for vulvovaginal candidiasis. Patient Prefer Adherence 2013:8:31-4. doi:10.2147/PPA.S38984

6 Palmsten K, Hernández-Díaz S, Chambers CD, et al. The most commonly dispensed prescription medications among pregnant women enrolled in the US Medicaid program. Obstet Gynecol 2015:126:465-73 doi:10.1097/AOG.0000000000000982

7 Tiboni GM, Giampietro F. Murine teratology of fluconazole: evaluation of developmental phase specificity and dose dependence. Pediatr Res 2005:58:94-9. doi:10.1203/01.PDR.0000166754.24957.73

8 Tiboni GM. Second branchial arch anomalies induced by fluconazole, a bis-triazole antifungal agent, in cultured mouse embryos. Res Commun Chem Pathol Pharmacol 1993;79:381-4

9 Menegola E, Broccia ML, Di Renzo F, Giavini E. Pathogenic pathways in fluconazole-induced branchial arch malformations. Birth Defects Res A Clin Mol Teratol 2003;67:116-24. doi:10.1002/bdra.10022

10 Menegola E, Broccia ML, Di Renzo F, Giavini E. Antifungal triazoles induce malformations in vitro. Reprod Toxicol 2001;15:421-7. doi:10.1016/S0890-6238(01)00143-5

11 Lee BE, Feinberg M, Abraham JJ, Murthy AR. Congenital malformations in an infant born to a woman treated with fluconazole. Pediatr Infect Dis / 1992;11:1062-4. doi:10.1097/00006454-199211120-00017

12 Pursley TJ, Blomquist IK, Abraham J, Andersen HF, Bartley JA Fluconazole-induced congenital anomalies in three infants. Clin Infect Dis 1996;22:336-40. doi:10.1093/clinids/22.2.336

13 Aleck KA, Bartley DL. Multiple malformation syndrome following fluconazole use in pregnancy: report of an additional patient. Am J Med Genet 1997;72:253-6. doi:10.1002/(SICI)10968628(19971031)72:3<253::AID-AJMG1>3.0.CO;2-S

14 Jick SS. Pregnancy outcomes after maternal exposure to fluconazole. Pharmacotherapy 1999;19:221-2. doi:10.1592/ phco.19.3.221.30919

15 Sorensen HT, Nielsen GL, Olesen C, et al. Risk of malformations and other outcomes in children exposed to fluconazole in utero. Br J Clin Pharmacol 1999;48:234-8. doi:10.1046/j.13652125.1999.00989.x

16 Nørgaard M, Pedersen L, Gislum M, et al. Maternal use of fluconazole and risk of congenital malformations: a Danish population-based cohort study. J Antimicrob Chemother 2008;62:172-6. doi:10.1093/ $\mathrm{jac} / \mathrm{dkn} 157$

17 Mølgaard-Nielsen D, Pasternak B, Hviid A. Use of oral fluconazole during pregnancy and the risk of birth defects. N Engl J Med 2013:369:830-9. doi:10.1056/NEIMoa1301066

18 Howley MM, Carter TC, Browne ML, Romitti PA, Cunniff CM, Druschel CM, National Birth Defects Prevention Study. Fluconazole use and birth defects in the National Birth Defects Prevention Study. Am J Obstet Gynecol 2016;214:657.e1-9. doi:10.1016/j. ajog.2015.11.022.

19 Bérard A, Sheehy O, Zhao JP, et al. Associations between low- and highdose oral fluconazole and pregnancy outcomes: 3 nested case-control studies. CMAI 2019;191:E179-87. doi:10.1503/cmaj.180963

20 Liu D, Zhang C, Wu L, Zhang L, Zhang L. Fetal outcomes after maternal exposure to oral antifungal agents during pregnancy: A systematic review and meta-analysis. Int J Gynaecol Obstet 2020;148:6-13. doi:10.1002/ijgo.12993

21 Palmsten K, Huybrechts KF, Mogun H, et al. Harnessing the Medicaid Analytic eXtract (MAX) to evaluate medications in pregnancy: design considerations. PLoS One 2013;8:e67405. doi:10.1371/journal. pone.0067405

22 Knox CA, Hampp C, Palmsten K, et al. Validation of mother-infant linkage using Medicaid Case ID variable within the Medicaid
Analytic eXtract (MAX) database. Pharmacoepidemiol Drug Saf 2019:28:1222-30. doi:10.1002/pds.4843

23 Huybrechts KF, Palmsten K, Avorn J, et al. Antidepressant use in pregnancy and the risk of cardiac defects. N Engl I Med 2014;370:2397-407. doi:10.1056/NEJMoa1312828

24 Patorno E, Huybrechts KF, Bateman BT, et al. Lithium use in pregnancy and the risk of cardiac malformations. N Engl I Med 2017;376:2245-54. doi:10.1056/NEJMoa1612222

25 Bateman BT, Hernandez-Diaz S, Fischer MA, et al. Statins and congenital malformations: cohort study. BMJ 2015;350:h1035. doi:10.1136/bmj.h1035

26 Huybrechts KF, Bateman BT, Palmsten K, et al. Antidepressant use late in pregnancy and risk of persistent pulmonary hypertension of the newborn. JAMA 2015;313:2142-51. doi:10.1001/jama.2015.5605

27 Huybrechts KF, Hernández-Díaz S, Straub L, et al. Association of maternal first-trimester ondansetron use with cardiac malformations and oral clefts in offspring. JAMA 2018;320:2429-37. doi:10.1001/ jama.2018.18307

28 Bateman BT, Heide-Jørgensen U, Einarsdóttir K et al. $\beta$-blocker use in pregnancy and the risk for congenital malformations: an international cohort study. Ann Intern Med 2018;169:665-73. doi:10.7326/M18 0338

29 Palmsten K, Huybrechts KF, Mogun H, et al. Harnessing the Medicaid Analytic eXtract (MAX) to evaluate medications in pregnancy: design considerations. PLoS One 2013;8:e67405. doi:10.1371/journal. pone.0067405

30 Soong D, Einarson A. Vaginal yeast infections during pregnancy. Can Fam Physician 2009;55:255-6.

31 King CT, Rogers PD, Cleary JD, Chapman SW. Antifungal therapy during pregnancy. Clin Infect Dis 1998;27:1151-60. doi:10.1086/514977

32 Czeizel AE, Kazy Z, Puhó E. Population-based case-control teratologic study of topical miconazole. Congenit Anom (Kyoto) 2004;44:41-5. doi:10.1111/j.1741-4520.2004.00007x

33 Czeizel AE, Tóth M, Rockenbauer M. No teratogenic effect after clotrimazole therapy during pregnancy. Epidemiology 1999;10:43740. doi:10.1097/00001648-199907000-00016

34 Rotem R, Fishman B, Daniel S, Koren G, Lunenfeld E, Levy A. Risk of major congenital malformations following first-trimester exposure to vaginal azoles used for treating vulvovaginal candidiasis: a population-based retrospective cohort study. BJOG 2018;125:15506. doi:10.1111/1471-0528.15293

35 Cottreau JM, Barr VO. A review of antiviral and antifungal use and safety during pregnancy. Pharmacotherapy 2016;36:668-78. doi:10.1002/phar.1764

36 Giavini E, Menegola E. Are azole fungicides a teratogenic risk for human conceptus?Toxicol Lett 2010;198:106-11. doi:10.1016/j. toxlet.2010.07.005

37 Palmsten K, Huybrechts KF, Kowal MK, Mogun H, Hernández-Díaz S. Validity of maternal and infant outcomes within nationwide Medicaid data. Pharmacoepidemiol Drug Saf 2014;23:646-55. doi:10.1002/ pds. 3627

38 Cooper WO, Hernandez-Diaz S, Gideon P, et al. Positive predictive value of computerized records for major congenital malformations. Pharmacoepidemiol Drug Saf 2008;17:455-60. doi:10.1002/ pds. 1534

39 Peahl AF, Dalton VK, Montgomery IR, Lai YL, Hu HM, Waljee JF. Rates of new persistent opioid use after vaginal or cesarean birth among US women. JAMA Netw Open 2019;2:e197863. doi:10.1001/ jamanetworkopen.2019.7863

40 Bateman BT, Mhyre JM, Hernandez-Diaz S, et al. Development of a comorbidity index for use in obstetric patients. Obstet Gynecol 2013;122:957-65. doi:10.1097/AOG.0b013e3182a603bb

41 Schneeweiss S, Seeger JD, Maclure M, Wang PS, Avorn J, Glynn RJ. Performance of comorbidity scores to control fo confounding in epidemiologic studies using claims data. Am J Epidemiol 2001;154:854-64. doi:10.1093/aje/154.9.854

42 Desai RJ, Rothman KJ, Bateman BT, Hernandez-Diaz S, Huybrechts KF. A propensity-score-based fine stratification approach for confounding adjustment when exposure is infrequent. Epidemiology 2017:28:249-57 doi:10.1097/EDE 0000000000000595

43 Desai RJ, Franklin JM. Alternative approaches for confounding adjustment in observational studies using weighting based on the propensity score: a primer for practitioners. BM/ 2019;367:15657. doi:10.1136/bmj.15657

44 Lopez-Rangel E, Van Allen MI. Prenatal exposure to fluconazole: an identifiable dysmorphic phenotype. Birth Defects Res A Clin Mol Teratol 2005;73:919-23 doi:10.1002/bdra.20189

45 Mastroiacovo P, Mazzone T, Botto LD, et al. Prospective assessment of pregnancy outcomes after first-trimester exposure to fluconazole. Am J Obstet Gynecol 1996;175:1645-50. doi:10.1016/S00029378(96)70119-9

Web appendix: Supplementary material 